\title{
The Thai version of the Juvenile Arthritis Multidimensional Assessment Report (JAMAR)
}

\author{
Soamarat Vilaiyuk ${ }^{1}$. Sirisucha Soponkanaporn ${ }^{1} \cdot$ Butsabong Lerkvaleekul $^{1}$. Tapanee Pipatkullachart ${ }^{1}$. \\ Dara Mairiang ${ }^{1}$ - Alessandro Consolaro ${ }^{2,3}$. Francesca Bovis ${ }^{2} \cdot$ Nicolino Ruperto $^{2}$. For the Paediatric Rheumatology \\ International Trials Organisation (PRINTO)
}

Received: 22 December 2017 / Accepted: 11 January 2018

(c) The Author(s) 2018. This article is an open access publication

\begin{abstract}
The Juvenile Arthritis Multidimensional Assessment Report (JAMAR) is a new parent/patient-reported outcome measure that enables a thorough assessment of the disease status in children with juvenile idiopathic arthritis (JIA). We report the results of the cross-cultural adaptation and validation of the parent and patient versions of the JAMAR in the Thai language. The reading comprehension of the questionnaire was tested in ten JIA parents and patients. Each participating centre was asked to collect demographic, clinical data and the JAMAR in 100 consecutive JIA patients or all consecutive patients seen in a 6-month period and to administer the JAMAR to 100 healthy children and their parents. The statistical validation phase explored descriptive statistics and the psychometric issues of the JAMAR: the three Likert assumptions, floor/ceiling effects, internal consistency, Cronbach's alpha, interscale correlations, test-retest reliability, and construct validity (convergent and discriminant validity). A total of 104 JIA patients (45.2\% systemic JIA, 10.6\% oligoarticular, 9.6\% RF negative polyarthritis, $34.6 \%$ other categories) and 102 healthy children, were enrolled in one paediatric rheumatology centre. Notably, none of the enrolled JIA patients is affected with psoriatic arthritis or undifferentiated arthritis. The JAMAR components discriminated well healthy subjects from JIA patients. All JAMAR components revealed satisfactory psychometric performances. In conclusion, the Thai version of the JAMAR is a valid tool for the assessment of children with JIA and is suitable for use both in routine clinical practice and clinical research.
\end{abstract}

Keywords Juvenile idiopathic arthritis · Disease status $\cdot$ Functional ability $\cdot$ Health-related quality of life $\cdot$ JAMAR

The local members of the Paediatric Rheumatology International
Trials Organisation (PRINTO) participating in the project
are listed in the dedicated tables no. 2 and 3 of "https://doi.
org/10.1007/s00296-018-3944-1 / Cross-cultural adaptation
and psychometric evaluation of the Juvenile Arthritis
Multidimensional Assessment Report (JAMAR) in 54 languages
across 52 countries: review of the general methodology".

Soamarat Vilaiyuk

soamarat21@hotmail.com

Nicolino Ruperto

nicolaruperto@gaslini.org

https://www.printo.it

Sirisucha Soponkanaporn

sirisuchars@yahoo.com

Butsabong Lerkvaleekul

lekky_med@hotmail.com

Tapanee Pipatkullachart

beauideak@gmail.com

Dara Mairiang

dowdara@gmail.com
Alessandro Consolaro

alessandroconsolaro@gaslini.org

Francesca Bovis

francescabovis@gaslini.org

1 Rheumatology Division, Pediatric Department, Faculty of Medicine Ramathibodi Hospital, Mahidol University, 270 Rama VI road, Bangkok 10400, Thailand

2 Clinica Pediatrica e Reumatologia, Paediatric Rheumatology International Trials Organisation (PRINTO), Istituto Giannina Gaslini, Via Gaslini 5, 16147 Genoa, Italy

3 Dipartimento di Pediatria, Università di Genova, Genoa, Italy 


\section{Introduction}

The aim of the present study was to cross-culturally adapt and validate the Thai parent, child/adult version of the Juvenile Arthritis Multidimensional Assessment Report (JAMAR) [1] in patients with juvenile idiopathic arthritis (JIA). The JAMAR assesses the most relevant parent/ patient-reported outcomes in JIA, including overall wellbeing, functional status, health-related quality of life (HRQoL), pain, morning stiffness, disease activity/status/ course, articular and extra-articular involvement, drugrelated side effects/compliance and satisfaction with illness outcome.

This project was part of a larger multinational study conducted by the Paediatric Rheumatology International Trials Organisation (PRINTO) [2] aimed to evaluate the epidemiology, outcome and treatment of childhood arthritis (EPOCA) in different geographic areas [3].

We report herein the results of the cross-cultural adaptation and validation of the parent and patient versions of the JAMAR in the Thai language.

\section{Materials and methods}

The methodology employed has been described in detail in the introductory paper of the supplement [4]. In brief, it was a cross-sectional study of JIA children, classified according to the ILAR criteria $[5,6]$ and enrolled from June 2015 to November 2015. Children were recruited after Ethics Committee approval and consent from at least one parent.

\section{The JAMAR}

The JAMAR [1] includes the following 15 sections.

1. Assessment of physical function (PF) using 15-items in which the ability of the child to perform each task is scored as follows: $0=$ without difficulty, $1=$ with some difficulty, 2 = with much difficulty, $3=$ unable to do and not applicable if it was not possible to answer the question or the patient was unable to perform the task due to their young age or to reasons other than JIA. The total PF score ranges from 0 to 45 and has three components: PF-lower limbs (PF-LL); PF-hand and wrist (PF-HW) and PF-upper segment (PF-US) each scoring from 0 to 15 [7]. Higher scores indicating higher degree of disability [8-10].

2. Rating of the intensity of the patient's pain on a 21-numbered circle Visual Analogue Scale (VAS) [11].
3. Assessment of the presence of joint pain or swelling (present/absent for each joint).

4. Assessment of morning stiffness (present/absent).

5. Assessment of extra-articular symptoms (fever and rash) (present/absent).

6. Rating of the level of disease activity on a 21-circle VAS.

7. Rating of disease status at the time of the visit (categorical scale).

8. Rating of disease course from previous visit (categorical scale).

9. Checklist of the medications the patient is taking (list of choices).

10. Checklist of side effects of medications.

11. Report of difficulties with medication administration (list of items).

12. Report of school/university/work problems caused by the disease (list of items).

13. Assessment of HRQoL, through the physical health $(\mathrm{PhH})$, and psychosocial health $(\mathrm{PsH})$ subscales (five items each) and a total score. The four-point Likert response, referring to the prior month, are 'never' (score $=0)$, 'sometimes' (score $=1$ ), 'most of the time' $($ score $=2)$ and 'all the time' ( able' column was included in the parent version of the questionnaire to designate questions that cannot be answered because of developmental immaturity. The total HRQoL score ranges from 0 to 30 , with higher scores indicating worse HRQoL.

A separate score for PhH and PsH (range 0-15) can be calculated [12-14].

14. Rating of the patient's overall well-being on a 21-numbered circle VAS.

15. A question about satisfaction with the outcome of the illness (yes/no) [15].

The JAMAR is available in three versions, one for parent proxy-report (child's age 2-18), one for child self-report, with the suggested age range of 7-18 years, and one for adults.

\section{Cross-cultural adaptation and validation}

The process of cross-cultural adaptation was conducted according to international guidelines with 2-3 forward and backward translations. In those countries for which the translation of JAMAR had been already cross-cultural adapted in a similar language (i.e. Spanish in South American countries), only the probe technique was performed. Reading comprehension and understanding of the translated 
questionnaires were tested in a probe sample of ten JIA parents and ten patients.

Each participating centre was asked to collect demographic, clinical data and the JAMAR in 100 consecutive JIA patients or all consecutive patients seen in a 6-month period and to administer the JAMAR to 100 healthy children and their parents.

The statistical validation phase explored the descriptive statistics and the psychometric issues [16]. In particular, we evaluated the following validity components: the first Likert assumption (mean and standard deviation [SD] equivalence); the second Likert assumption or equal items-scale correlations (Pearson $r$ all items within a scale should contribute equally to the total score); third Likert assumption (item internal consistency or linearity for which each item of a scale should be linearly related to the total score that is $90 \%$ of the items should have Pearson $r \geq 0.4$ ); floor/ceiling effects (frequency of items at lower and higher extremes of the scales, respectively); internal consistency, measured by the Cronbach's alpha, interscale correlation (the correlation between two scales should be lower than their reliability coefficients, as measured by Cronbach's alpha); test-retest reliability or intra-class correlation coefficient (reproducibility of the JAMAR repeated after 1 or 2 weeks); and construct validity in its two components: the convergent or external validity which examines the correlation of the JAMAR subscales with the six JIA core set variables, with the addition of the parent assessment of disease activity and pain by the Spearman's correlation coefficients $(r)$ [17] and the discriminant validity, which assesses whether the JAMAR discriminates between the different JIA categories and healthy children [18].

Quantitative data were reported as medians with 1st and 3 rd quartiles and categorical data as absolute frequencies and percentages.

The complete Thai parent and patient versions of the JAMAR are available upon request to PRINTO.

\section{Results}

\section{Cross-cultural adaptation}

The Thai JAMAR was fully cross-culturally adapted with two forward and two backward translations. The concordance rate between the original standard English version of the JAMAR and the two back-translations was $68.3 \%$ $(84 / 123$ lines) for the parent version and $70.8 \%(85 / 120$ lines) for the child version.

Of the 123 lines in the patient version of the JAMAR, $119(97 \%)$ lines were understood by at least $80 \%$ of the ten parents tested $($ median $=100 \%$; range $60-100 \%)$. Of the 120 lines in the patient version of the JAMAR, 116
(97\%) lines were understood by at least $80 \%$ of the children (median $=100 \%$; range $40-100 \%$ ). Lines 47, 60, 111, and 114 of the parent version of the JAMAR and lines 45,58 , 108 , and 111 of the child version of the JAMAR were modified considering to parents' and patients' suggestions.

\section{Demographic and clinical characteristics of the subjects}

A total of 104 JIA patients and 102 healthy children (total of 206 subjects), were enrolled at the paediatric rheumatology clinic in Ramathibodi Hospital.

In the 104 JIA subjects, the JIA categories were $45.2 \%$ with systemic JIA, $10.6 \%$ with oligoarthritis, $9.6 \%$ with RF negative polyarthritis, $10.6 \%$ with RF positive polyarthritis and $24.0 \%$ with enthesitis related arthritis. Notably, none of the enrolled JIA patients is affected with psoriatic arthritis or undifferentiated arthritis (Table 1).

A total of 188/206 (91.3\%) subjects had the parent version of the JAMAR completed by a parent ( 87 from parents of JIA patients and 101 from parents of healthy children). The JAMAR was completed by $168 / 188(89.4 \%)$ mothers and 20/188 (10.6\%) fathers. The child version of the JAMAR was completed by 141/206 (68.4\%) children age 5.1 or older. Also patients younger than 7 year old, capable to assess their personal condition and able to read and write, were asked to fill in the patient version of the questionnaire.

\section{Discriminant validity}

The JAMAR results are presented in Table 1, including the scores [median ( $1^{\text {st }}-3$ rd quartile $)$ ] obtained for the PF, the $\mathrm{PhH}$, the PsH subscales and total score of the HRQoL scales. The JAMAR components discriminated well between healthy subjects and JIA patients.

In summary, the JAMAR revealed that JIA patients had a greater level of disability and pain, as well as a lower HRQoL than their healthy peers.

\section{Psychometric issues}

The main psychometric properties of both parent and child versions of the JAMAR are reported in Table 2. The following "Results" section refers mainly to the parent's version findings, unless otherwise specified.

\section{Descriptive statistics (first Likert assumption)}

There were no missing results for all JAMAR items, since data were collected through a web-based system that did not allow to skip answers and input of null values. The response pattern for both PF and HRQoL was positively skewed toward normal functional ability and normal 


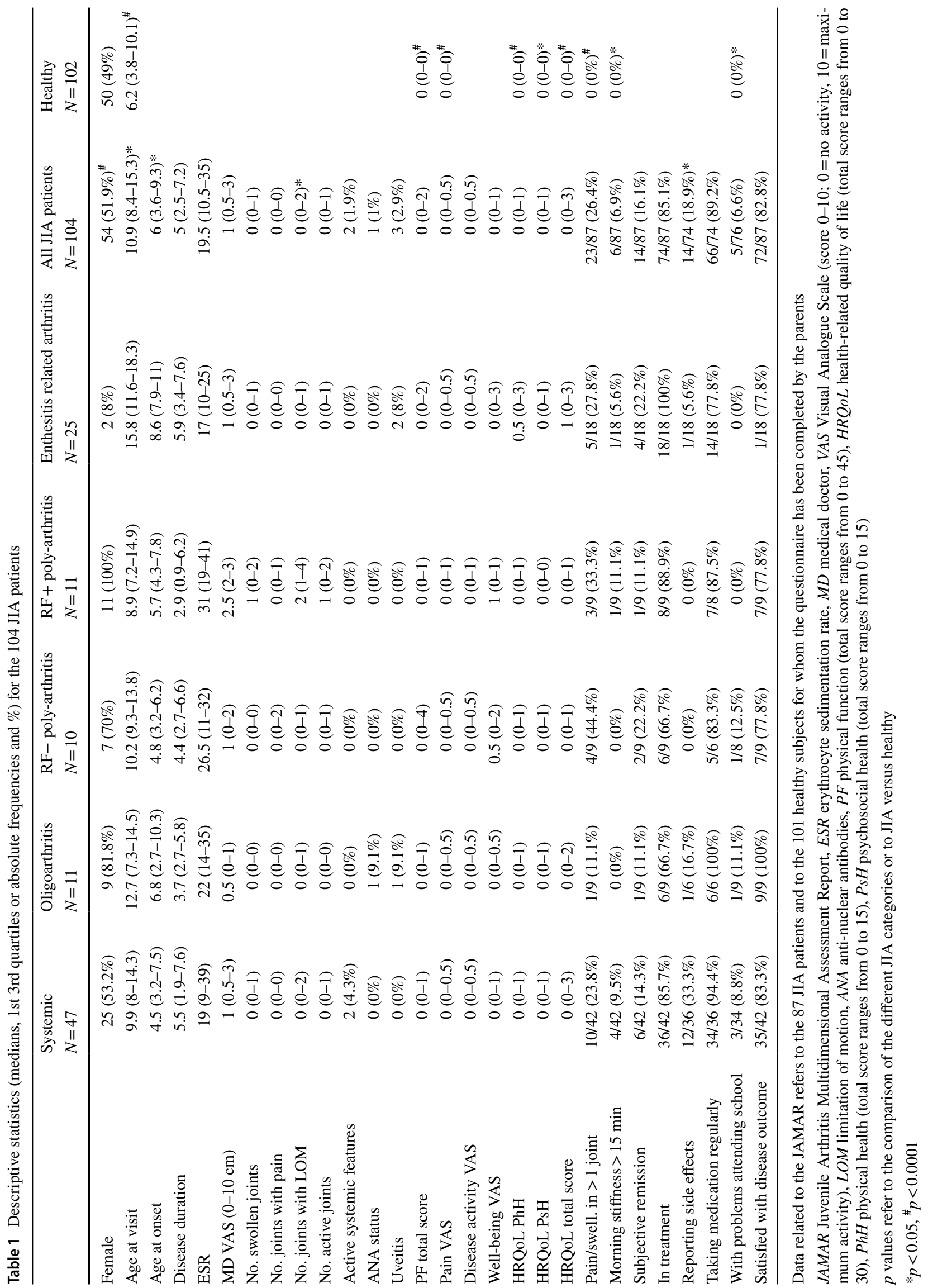


Table 2 Main psychometric characteristics between the parent and child version of the JAMAR

\begin{tabular}{|c|c|c|}
\hline & Parent $N=87 / 188$ & Child $N=92 / 141$ \\
\hline Missing values (1st-3rd quartiles) & No missing values & No missing values \\
\hline Response pattern & PF and HRQoL positively skewed & PF and HRQoL positively skewed \\
\hline \multicolumn{3}{|l|}{ Floor effect, median } \\
\hline $\mathrm{PF}$ & $94.3 \%$ & $94.6 \%$ \\
\hline HRQoL PhH & $80.5 \%$ & $89.1 \%$ \\
\hline HRQoL PsH & $87.4 \%$ & $89.1 \%$ \\
\hline Pain VAS & $69.0 \%$ & $69.6 \%$ \\
\hline Disease activity VAS & $66.7 \%$ & $67.4 \%$ \\
\hline Well-being VAS & $56.3 \%$ & $65.2 \%$ \\
\hline \multicolumn{3}{|l|}{ Ceiling effect, median } \\
\hline $\mathrm{PF}$ & $0.0 \%$ & $0.0 \%$ \\
\hline HRQoL PhH & $1.1 \%$ & $0.0 \%$ \\
\hline HRQoL PsH & $0.0 \%$ & $0.0 \%$ \\
\hline Pain VAS & $0.0 \%$ & $0.0 \%$ \\
\hline Disease activity VAS & $0.0 \%$ & $0.0 \%$ \\
\hline Well-being VAS & $0.0 \%$ & $0.0 \%$ \\
\hline Items with equivalent item-scale correlation & $67 \%$ for $\mathrm{PF}, 90 \%$ for $\mathrm{HRQoL}$ & $87 \%$ for $\mathrm{PF}, 80 \%$ for $\mathrm{HRQoL}$ \\
\hline Items with items-scale correlation $\geq 0.4$ & $73 \%$ for $\mathrm{PF}, 70 \%$ for $\mathrm{HRQoL}$ & $80 \%$ for PF, $80 \%$ for $\mathrm{HRQoL}$ \\
\hline \multicolumn{3}{|l|}{ Cronbach's alpha } \\
\hline PF-LL & 0.84 & 0.77 \\
\hline PF-HW & 0.76 & 0.85 \\
\hline PF-US & 0.60 & 0.74 \\
\hline HRQoL-PhH & 0.77 & 0.77 \\
\hline HRQoL-PsH & 0.65 & 0.73 \\
\hline Items with item-scale correlation lower than the Cronbach alpha & $87 \%$ for $\mathrm{PF}, 90 \%$ for $\mathrm{HRQoL}$ & $87 \%$ for $\mathrm{PF}, 100 \%$ for $\mathrm{HRQoL}$ \\
\hline \multicolumn{3}{|l|}{ Test-retest intraclass correlation } \\
\hline PF total score & 0.96 & 0.80 \\
\hline HRQoL-PhH & 0.95 & 0.57 \\
\hline HRQoL-PsH & 0.00 & 0.00 \\
\hline \multicolumn{3}{|l|}{ Spearman's correlation with JIA core-set variables, median } \\
\hline $\mathrm{PF}$ & 0.4 & 0.4 \\
\hline HRQoL PhH & 0.6 & 0.6 \\
\hline HRQoL PsH & 0.2 & 0.2 \\
\hline Pain VAS & 0.4 & 0.4 \\
\hline Disease activity VAS & 0.4 & 0.5 \\
\hline Well-being VAS & 0.4 & 0.4 \\
\hline
\end{tabular}

JAMAR Juvenile Arthritis Multidimensional Assessment Report, JIA juvenile idiopathic arthritis, VAS Visual Analogue Scale, $P F$ physical function, $H R Q o L$ health-related quality of life, $P h H$ physical health, $P s H$ psychosocial health, $P F-L L$ PF-lower limbs, $P F-H W$ PF-hand and wrist, $P F$-US PF-upper segment

HRQoL. A reduced number of response choices were used for the different HRQoL items except for items 2, 3 and 4, whereas a reduced number of response choices was used for all the PF items except for items 3 and 5. The mean and $\mathrm{SD}$ of the items within a scale were roughly equivalent for the PF and for the HRQoL items (data not shown). The median number of items marked as not applicable was $0 \%$ $(0-1 \%)$ for the PF and $0 \%(0-1 \%)$ for the HRQoL.

\section{Floor and ceiling effect}

The median floor effect was $94.3 \%$ (88.5-96.6\%) for the PF items, $80.5 \%$ (77.0-90.8\%) for the HRQoL PhH items, and 87.4\% (86.2-94.3\%) for the HRQoL PsH items. The median ceiling effect was $0 \%(0-1.1 \%)$ for the PF items, $1.1 \%$ $(0-2.3 \%)$ for the HRQoL PhH items, and $0.0 \%(0-0.0 \%)$ for the HRQoL PsH items. The median floor effect was $69.0 \%$ for the pain VAS, $66.7 \%$ for the disease activity VAS and 
$56.3 \%$ for the well-being VAS. The median ceiling effect was $0 \%$ for the pain VAS, $0 \%$ for the disease activity VAS and $0 \%$ for the well-being VAS.

\section{Equal items-scale correlations (second Likert assumption)}

Pearson items-scale correlations corrected for overlap were roughly equivalent for items within a scale for $67 \%$ of the PF items, with the exception of PF items 6, 10, 11, 14 and 15 , and for $90 \%$ of the HRQoL items, with the exception of HRQoL item 1.

\section{Items internal consistency (third Likert assumption)}

Pearson items-scale correlations were $\geq 0.4$ for $73 \%$ of items of the PF (except for PF items 10, 11, 14 and 15) and 70\% of items of the HRQoL (except for HRQoL items 1, 7 and 9).

\section{Cronbach's alpha internal consistency}

Cronbach's alpha was 0.84 for PF-LL, 0.76 for PF-HW, 0.60 for PF-US. Cronbach's alpha was 0.77 for HRQoL-PhH and 0.65 for HRQoL-PsH.

\section{Interscale correlation}

The Pearson correlation of each item of the PF and the HRQoL with all items included in the remaining scales of the questionnaires was lower than the Cronbach's alpha except for the PF items 12 and 13, and for the HRQoL item 4.

\section{Test-retest reliability}

Reliability was assessed in eight JIA patients, by re-administering both versions (parent and child) of the JAMAR after a median of 8 days ( $8-8$ days). The intraclass correlation coefficients (ICC) for the PF total score showed an almost perfect reproducibility $(\mathrm{ICC}=0.96)$. The ICC for the HRQoL PhH showed an almost perfect reproducibility $(\mathrm{ICC}=0.95)$ while the ICC for the HRQoL PsH showed a poor reproducibility $(\mathrm{ICC}=0.0)$.

\section{Convergent validity}

The Spearman's correlation of the PF total score with the JIA core set of outcome variables ranged from 0.3 to 0.5 (median $=0.4)$. The PF total score best correlation was observed with the parent assessment of pain $(r=0.5$, $p<0.001)$. The correlation of the PF total score with the ESR was not significant $(p=0.17)$. For the HRQoL, the median correlation of the $\mathrm{PhH}$ with the JIA core set of outcome variables ranged from 0.2 to 0.7 (median $=0.6$ ), whereas for the $\mathrm{PsH}$ ranged from 0.04 to 0.4 (median $=0.2$ ). The $\mathrm{PhH}$ showed the best correlation with the parent's assessment of pain $(r=0.7, p<0.001)$ and the $\mathrm{PsH}$ with the physician global assessment of well-being $(r=0.4, p=0.0001)$. The median correlations between the pain VAS, the well-being VAS, and the disease activity VAS and the physician-centred and laboratory measures were $0.4(0.3-0.5), 0.4(0.2-0.5)$, $0.4(0.3-0.4)$, respectively.

\section{Discussion}

In this study, the Thai version of the JAMAR was fully crossculturally adapted from the original standard English version with two forward and two backward translations. According to the results of the validation analysis, the Thai parent and patient versions of the JAMAR possess satisfactory psychometric properties. The disease-specific components of the questionnaire discriminated well between patients with JIA and healthy controls.

Psychometric performances were good for all domains of the JAMAR with some exceptions: four PF items ("open and close a tap or open a previously open jar", "stretch out arms", "bend head back" and "bite a sandwich or an apple") and three HRQoL items ("have difficulty taking care", "feeling nervous or anxious" and "have difficulty concentrating or paying attention") showed a lower items internal consistency. Furthermore, the overall internal consistency for PF-US and for HRQoL-PsH subscales were questionable.

In the external validity evaluation, the Spearman's correlations of the PF and HRQoL scores with JIA core set parameters ranged from weak to strong.

The results obtained for the parent version of the JAMAR are very similar to those obtained for the child version, which suggests that children are equally reliable proxy reporters of their disease and health status as their parents. The JAMAR is aimed to evaluate the side effects of medications and school attendance, which are other dimensions of daily life that were not previously considered by other HRQoL tools. This may provide useful information for intervention and follow-up in health care. In conclusion, the Thai version of the JAMAR was found to have satisfactory psychometric properties and it is, thus, a reliable and valid tool for the multidimensional assessment of children with JIA.

Acknowledgements We thank all families who participated in the project, the team that prepared and reviewed the forward and backward translations, and all members of PRINTO in Thailand. We thank Surintra Vilaiyuk, MD, Chongchit Soponkanaporn, and Mahippathorn Chinnapha, MD for the translation process, Kanyaphak Phumeesri, Jutamas Saentaweesuk, and Sasivimon Unchanbutr for data collection, Paramee Jarupan and Supanee Treepetchkul for data entry. We thank the staff of the PRINTO International Coordinating Centre in Genoa 
(Italy) and in particular Marco Garrone for the overall coordination of the translation process, Silvia Scala and Elisa Patrone for data collection and quality assurance, Luca Villa, Giuseppe Silvestri and Mariangela Rinaldi for the database development and management and the remaining PRINTO team for data entry. The Principal Investigator of the study was Prof. Angelo Ravelli, MD. The scientific coordinator and study methodologist was Nicolino Ruperto, MD, MPH. The project coordinators were Alessandro Consolaro, MD, PhD, Francesca Bovis, BsA. We also thank Prof. Alberto Martini, PRINTO Chairman. Funding was provided by the Istituto G. Gaslini, Genoa (Italy). Permission for the use of JAMAR and its translations must be obtained in writing from PRINTO, Genoa, Italy. All JAMAR-related inquiries should be directed to at printo@ gaslini.org. Permission for the use of CHAQ and CHQ derived-material is granted through the scientific cooperation of the copyright holder ICORE of Woodside CA and HealthActCHQ Inc. of Boston, Massachusetts USA. All CHQ-related inquiries should be directed to licensing@ healthactchq.com. All CHAQ-related inquiries should be directed to gsingh@stanford.edu.

Funding This study was funded and coordinated by Istituto Giannina Gaslini, Genoa, Italy.

\section{Compliance with Ethical Standards}

Conflict of interest Dr. Vilaiyuk reports funding support from Istituto Giannina Gaslini, Genoa, Italy, for the data collection and translation performed at her site within the EPOCA project. Dr. Ruperto has received grants from BMS, Hoffman-La Roche, Janssen, Novartis, Pfizer, Sobi, during the conduct of the study and personal fees and speaker honorarium from Abbvie, Ablynx, Amgen, AstraZeneca, Baxalta Biosimilars, Biogen Idec, Boehringer, Bristol Myers Squibb, Celgene, EliLilly, EMD Serono, Gilead Sciences, Janssen, Medimmune, Novartis, Pfizer, Rpharm, Roche, Sanofi, Servier and Takeda. Dr. Consolaro, Dr. Bovis, Dr. Lerkvaleekul, Dr. Mairiang, Dr. Pipatkullachart and Dr. Soponkanaporn have nothing to disclose.

Ethical approval All procedures performed in studies involving human participants were in accordance with the ethical standards of the institutional and/or national research committee and with the 1964 Helsinki Declaration and its later amendments or comparable ethical standards.

Informed consent Informed consent was obtained from all individual participants included in the study as per the requirement of the local ethical committee.

Open Access This article is distributed under the terms of the Creative Commons Attribution 4.0 International License (http://creativeco mmons.org/licenses/by/4.0/), which permits unrestricted use, distribution, and reproduction in any medium, provided you give appropriate credit to the original author(s) and the source, provide a link to the Creative Commons license, and indicate if changes were made.

\section{References}

1. Filocamo G, Consolaro A, Schiappapietra B, Dalpra S, Lattanzi B, Magni-Manzoni S et al (2011) A new approach to clinical care of juvenile idiopathic arthritis: the Juvenile Arthritis Multidimensional Assessment Report. J Rheumatol 38(5):938-53

2. Ruperto N, Martini A (2011) Networking in paediatrics: the example of the Paediatric Rheumatology International Trials Organisation (PRINTO). Arch Dis Child 96(6):596-601

3. Consolaro A, Ruperto N, Filocamo G, Lanni S, Bracciolini G, Garrone M et al (2012) Seeking insights into the EPidemiology, treatment and outcome of childhood arthritis through a multinational collaborative effort: Introduction of the EPOCA study. Pediatr Rheumatol Online J 10(1):39

4. Bovis F, Consolaro A, Pistorio A, Garrone M, Scala S, Patrone E et al (2018) Cross-cultural adaptation and psychometric evaluation of the Juvenile Arthritis Multidimensional Assessment Report (JAMAR) in 54 languages across 52 countries: review of the general methodology. Rheumatol Int. https://doi.org/10.1007/ s00296-018-3944-1 (in this issue)

5. Petty RE, Southwood TR, Baum J, Bhettay E, Glass DN, Manners P et al (1998) Revision of the proposed classification criteria for juvenile idiopathic arthritis: Durban, 1997. J Rheumatol 25(10):1991-1994

6. Petty RE, Southwood TR, Manners P, Baum J, Glass DN, Goldenberg J et al (2004) International League of Associations for Rheumatology classification of juvenile idiopathic arthritis: second revision, Edmonton, 2001. J Rheumatol 31(2):390-392

7. Filocamo G, Sztajnbok F, Cespedes-Cruz A, Magni-Manzoni S, Pistorio A, Viola S et al (2007) Development and validation of a new short and simple measure of physical function for juvenile idiopathic arthritis. Arthritis Rheum 57(6):913-20

8. Lovell DJ, Howe S, Shear E, Hartner S, McGirr G, Schulte M et al (1989) Development of a disability measurement tool for juvenile rheumatoid arthritis. The juvenile arthritis functional assessment scale. Arthritis Rheum 32:1390-1395

9. Howe S, Levinson J, Shear E, Hartner S, McGirr G, Schulte M et al (1991) Development of a disability measurement tool for juvenile rheumatoid arthritis. The juvenile arthritis functional assessment report for children and their parents. Arthritis Rheum 34:873-80

10. Singh G, Athreya BH, Fries JF, Goldsmith DP (1994) Measurement of health status in children with juvenile rheumatoid arthritis. Arthritis Rheum 37:1761-1769

11. Filocamo G, Davi S, Pistorio A, Bertamino M, Ruperto N, Lattanzi B et al (2010) Evaluation of 21-numbered circle and 10-centimeter horizontal line visual analog scales for physician and parent subjective ratings in juvenile idiopathic arthritis. J Rheumatol 37(7):1534-1541

12. Duffy CM, Arsenault L, Duffy KN, Paquin JD, Strawczynski H (1997) The Juvenile Arthritis Quality of Life Questionnairedevelopment of a new responsive index for juvenile rheumatoid arthritis and juvenile spondyloarthritides. J Rheumatol 24(4):738-46

13. Varni JW, Seid M, Knight TS, Burwinkle T, Brown J, Szer IS (2002) The PedsQL (TM) in pediatric rheumatology—reliability, validity, and responsiveness of the pediatric quality of life inventory (TM) generic core scales and rheumatology module. Arthritis Rheum 46(3):714-25

14. Landgraf JM, Abetz L, Ware JE (1996) The CHQ user's manual, 1st edn. The Health Institute, New England Medical Center, Boston, MA

15. Filocamo G, Consolaro A, Schiappapietra B, Ruperto N, Pistorio A, Solari N et al (2012) Parent and child acceptable symptom state in juvenile idiopathic arthritis. J Rheumatol 39(4):856-63

16. Nunnally JC (1978) Psychometric theory, 2nd edn. McGraw-Hill, New York, NY

17. Giannini EH, Ruperto N, Ravelli A, Lovell DJ, Felson DT, Martini A (1997) Preliminary definition of improvement in juvenile arthritis. Arthritis Rheum 40(7):1202-1209

18. Ware JE Jr, Harris WJ, Gandek B, Rogers BW, Reese PR (1997) MAP-R for windows: multitrait/multi-item analysis programrevised user's guide. Version 1.0 ed. Health Assessment Lab, Boston, MA 